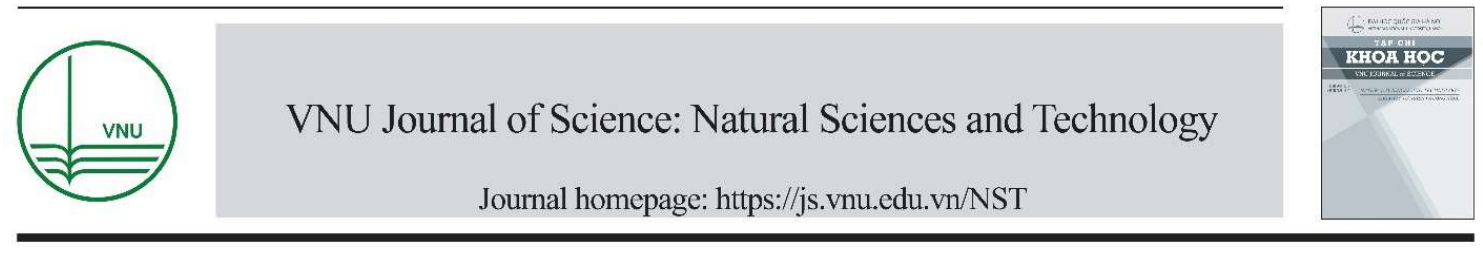

Original Article

\title{
Chemical Composition of Essential Oils from the Leaves of Syzygium Bullockii and Syzygium Tsoongii in Ke Go Nature Reserve, Ha Tinh Province
}

\author{
Tran Hau Khanh ${ }^{1,2, *}$, Pham Hong Ban ${ }^{1}$, Tran Minh $\mathrm{Hoi}^{3}$ \\ ${ }^{I}$ Vinh University, 182 Le Duan street, Vinh city, Nghe An Province, Vietnam \\ ${ }^{2}$ Ha Tinh Department of Science and Technology, 142 Tran Phu street, Ha Tinh city \\ ${ }^{3}$ Vietnam Academy of Science and Technology, 18 Hoang Quoc Viet, Cau Giay, Hanoi
}

Received 13 February 2020

Revised 24 July 2020; Accepted 30 July 2020

\begin{abstract}
Chemical composition of essential oils obtained from the leaf of Syzygium bullockii and Syzygium tsoongii were reported, corresponding oil content $0,36 \%$ and $0,27 \%$. The analysis was performed by means of gas chromatography-flame ionization detector (GC-FID) and gas chromatography coupled with mass spectrometry (GC-MS). Twenty-seven components were identified in leaf oil of Syzygium bullockii, which represented about $76,75 \%$ of the total composition of the oil and six components were not identified, which represented $15,72 \%$. The major constituents of the essential oil were (E)-caryophyllene (49,65\%), spathulenol (4,29\%), caryophyllene oxide $(4,14 \%)$, bicyclogermacrene (3,35\%), 2-tridecanone (3,25\%). Fourty-six components were identified in leaf oil of Syzygium tsoongii, which presented about $99.71 \%$ of the total composition of the oil. The major constituents of the essential oil were E-caryophyllene (23,40\%), bicyclogermacrene $(21,23 \%)$, (Z) $-\beta$ ocimene $(10,61 \%)$, $\alpha$-humulene $(6,33 \%)$, (E)- $\beta$-ocimene $(4,99 \%)$. For the first time, the chemical compositions of the essential oils of Syzygium bullockii and Syzygium tsoongii from Vietnam were being reported.
\end{abstract}

Keywords: Syzygium bullockii, Syzygium tsoongii, Myrtaceae, Ke Go, Ha Tinh.

${ }^{*}$ Corresponding author.

Email address: tranhaukhanh@gmail.com

https://doi.org/10.25073/2588-1140/vnunst.4999 


\title{
Thành phần hóa học tinh dầu từ lá loài Trâm bullock (Syzygium bullockii) và loài Trâm quả trắng (Syzygium tsoongii) ở Khu Bảo tồn thiên nhiên Kẻ Gỗ, tỉnh Hà Tĩnh
}

\author{
Trần Hậu Khanh ${ }^{1,2,}$, Phạm Hồng Ban ${ }^{1}$, Trần Minh Hợi ${ }^{3}$ \\ ${ }^{1}$ Trưòng Đại học Vinh, 182 Le Duẩn, Thành phố Vinh, Nghệ An \\ ${ }^{2}$ Sở Khoa hoc và Công nghệ Hà Tĩnh, 182 Trần Phú, Hà Tĩnh \\ ${ }^{3}$ Viện Hàn lâm Khoa học và Công nghệ Việt Nam, 18 Hoàng Quốc Việt, Cà̀u Giấy, Hà Nội \\ Nhận ngày 13 tháng 2 năm 2020 \\ Chỉnh sửa ngày 24 tháng 7 năm 2020; Chấp nhận đăng ngày 30 tháng 7 năm 2020
}

\begin{abstract}
Tóm tắt: Nghiên cứu thành phần hóa học tinh dầu từ lá loài Trâm bullock (Syzygium bullockii) và loài Trâm quả trắng (Syzygium tsoongii). Hàm lượng tinh dầu đạt lần lượt là $0,36 \%$ và $0,27 \%$ tương ứng theo nguyên liệu tươi. Tinh dầu được phân tích bằng sắc ký khí (GC) và sắc ký khí khối phổ (GC/MS). 27 hợp chất được xác định chiếm $76,75 \%$, còn 6 hợp chất chưa xác định tên chiếm $15,72 \%$ tồng lượng tinh dầu của loài Trâm bullock (Syzygium bullockii). Thành phần chính của tỉnh dầu là E-caryophyllene $(49,65 \%)$, spathulenol (4,29\%), caryophyllene oxide (4,14\%), bicyclogermacrene (3,35\%), 2-tridecanone (3,25\%). Loài Trâm suối (Syzygium tsoongii) đã xác định được 46 hợp chất chiếm $99,71 \%$ tổng lượng tinh dầu, Ecaryophyllene (23,40\%), bicyclogermacrene $(21,23 \%),(Z)-\beta$-ocimene $(10,61 \%), \alpha$-humulene $(6,33 \%)$, (E)$\beta$-ocimene $(4,99 \%)$ là các thành phần chính của tinh dầu. Đây là những dẫn liệu đầu tiên về hóa học tinh dầu của 2 loài này ở Việt Nam.
\end{abstract}

Tù khóa: Trâm bullock, Trâm quả trắng, họ Sim, Kẻ Gỗ, Hà Tĩnh.

\section{Mở đầu}

Chi Trâm trên thế giới có khoảng 1.200 loài phân bố ở các nước nhiệt đới và cận nhiệt đới [1-3]. Ở khu vực Đông Dương, tác giả Soh \& Parnell đã ghi nhận 27 loài phân bố ở Căm pu Chia, 30 loài ở Lào và 49 loài ở Việt Nam [4]. Ở Việt Nam, theo Nguyễn Tiến Bân, chi Trâm có 60 loài và 1 thứ [5]. Phạm Hoàng Hộ ghi nhận chi Trâm có 57 loài [6]. Một số loài được sử dụng làm thuốc [7-9]. Trâm bullock (Syzygium bullockii (Hance) Merr. \& Perry là cây bụi cao khoảng $1 \mathrm{~m}$, thường mọc trên đụn cát ven biển, có quả vào tháng 7-8, quả ăn được; mới thấy phân bố ở Thanh Hóa, Quảng Trị (Bến Hải), Quảng Bình, Thừa Thiên Huế (Phú Lộc), Đà Nẵng

\footnotetext{
*Tác giả liên hệ.

Địa chỉ email: tranhaukhanh@gmail.com

https://doi.org/10.25073/2588-1140/vnunst.4999
}

(Tourane). Còn có ở Trung Quốc (Hải Nam), Lào. Loài Trâm bullock (Syzygium bullockii (Hance) Merr. \& Perry) đã được tác giả Hance mô tả vào năm 1878 trên tạp chí "Journal of Botany" và nó được xếp vào chi Eugenia. Năm 1790 loài này được Lour mô tả và xếp vào chi Myrtus với tên khoa học Myrtus androsaemoides sensu Lour [5]. Trầm quả trắng (Syzygium tsoongii (Merr.) Merr. \& Perry) đã được tác giả Merry mô tả vào năm 1916 thuộc chi Eugenia có tên khoa học Eugenia leucocarpa Merry. Trâm quả trắng (Syzygium tsoongii (Merr.) Merr. \& Perry) là cây gỗ, mọc rải rác ven rừng thường xanh, dọc theo sông suối. Ra hoa tháng 10-12, có quả tháng 1-2; phân bố ở Quảng Ninh (Uông Bí, Hạ Long), Thanh Hóa, Quảng Bình, 
Quảng Trị (Bến Trạm), Đà Nẵng (Tourane), Nam Bộ. Còn có ở Trung Quốc (Quảng Đông, Hải Nam) [5]. Trên thế giới, một số loài trong chi như Syzygium polyanthum và Syzygium aromaticum đã được nghiên cứu về thành phần hóa học và hoạt tính kháng vi sinh vật của tinh dầu. Thành phần chính trong tinh dầu từ lá loài $S$. polyanthum là cis-4decanal (43,4\%), 1-decyl aldehyd (19,7\%), capryl aldehyd $(14,9 \%)$; thành phần chính trong tinh dầu từ lá loài $S$. aromaticum là p-eugenol $(75,1 \%)$, peugenol $(75,1 \%)$ và $\beta$-caryophyllene $(18,3 \%)$ [10]. Ở Việt Nam thành phần hóa học của tinh dầu và công dụng của một số loài cũng đã được nghiên cứu như Đinh hương (Syzygium aromaticum) với tinh dầu từ nụ hoa thì hợp chất eugenol (70-95\%), eugenyl acetat (1-5\%) và $\beta$-caryophyllene (4-12\%) và tinh dầu từ lá Gioi (Syzygium jambos): $\alpha$-pinene $(26,8 \% 0$, L-limonene $(23,8 \%)$ [11]. Thành phần hóa học chính trong tinh dầu lá của loài Syzygium hancei là $\gamma$-guaiene $(11.07 \%), \beta$-caryophyllene $(9.11 \%)$, cis-calamenene $(7.46 \%), \alpha$-copaene (6.97\%), trans-cadina-1,4-diene (5.09\%) [12]; tinh dầu từ lá của loài Syzygium caryophyllatum là $\beta$-caryophyllene $(42.53 \%)$, (E)- $\beta$-ocimene $(19.38 \%)$ và $\alpha$-humulene $(5.37 \%)$ và loài Syzygium lineatum là $\beta$-caryophyllene (64.53\%), $\alpha$-pinene $(6.14 \%), \alpha$-selinene $(4.76 \%)$ và $\alpha$ humulene $(4.39 \%)$ [12]. Nghiên cứu tinh dầu của hai loài này ở Việt Nam hiện chưa có bất kỳ công trình nào công bố. Bài báo này, là kết quả nghiên cứu thành phần hóa học tinh dầu của loài Trâm bullock (Syzygium bullockii) và Trâm quả trắng (Syzygium tsoongii) phân bố ở Khu Bảo tồn thiên nhiên Kẻ Gỗ, tỉnh Hà Tĩnh.

\section{Vật liệu và phương pháp nghiên cứu}

\subsection{Vật liệu}

Lá loài Trâm bullock (Syzygium bullockii) và Trâm quả trắng (Syzygium tsoongii) được thu hái ở Khu Bảo tồn thiên nhiên Kẻ Gố, tỉnh Hà Tĩnh vào tháng 7 năm 2019 (THK 811 và THK 807). Tiêu bản của hai loài này đã được định loại (Chuyên gia định loại: PGS.TS Phạm Hồng Ban - Đại học Vinh và PGS.TS Trần Minh Hợi - Viện Sinh thái và Tài nguyên Sinh vật) so với mẫu chuẩn và lưu giữ ở Bộ môn Thực vật, Viện Sư phạm Tự nhiên, trường Đại học Vinh.

\subsection{Phuoong pháp \\ + Tách tinh dầu}

Lá $(1 \mathrm{~kg})$ được cắt nhỏ và chưng cất bằng phương pháp lôi cuốn hơi nước trong thời gian 3 giờ ở áp suất thường theo dược điển Việt $\mathrm{Nam}$ IV [13].

\section{+ Phân tích tinh dầu}

Hoà tan $1,5 \mathrm{mg}$ tinh dầu đã được làm khô bằng $\mathrm{Na}_{2} \mathrm{SO}_{4}$ khan trong $1 \mathrm{ml} \mathrm{n}$-hexan tinh khiết loại dùng cho sắc kí và phân tích phổ.

Sắc kí khi $(G C)$ : Được thực hiện trên máy Agilent Technologies $\mathrm{HP} 6890 \mathrm{~N}$ Plus gắn vào detectơ FID của hãng Agilent Technologies, Mỹ. Cột sắc kí $\mathrm{HP}-5 \mathrm{MS}$ với chiều dài $30 \mathrm{~m}$, đường kính trong (ID) $=0,25 \mathrm{~mm}$, lớp phim mỏng $0,25 \mu \mathrm{m}$ đã được sử dụng. Khí mang $\mathrm{H}_{2}$. Nhiệt độ buồng bơm mẫu (Kĩ thuật chương trình nhiệt độPTV) $250^{\circ} \mathrm{C}$. Nhiệt độ Detectơ $260^{\circ} \mathrm{C}$. Chương trình nhiệt độ buồng điều nhiệt: $60^{\circ} \mathrm{C}(2$ phút), tăng $4^{\circ} \mathrm{C} /$ phút cho đến $220^{\circ} \mathrm{C}$, dừng ở nhiệt độ này trong 10 phút.

\section{Sắc ki khi-khối phổ (GC/MS):}

Việc phân tích định tính được thực hiện trên hệ thống thiết bị sắc kí khí và phổ kí liên hợp GC/MS của hãng Agilent Technologies HP $6890 \mathrm{~N}$. Agilent Technologies HP $6890 \mathrm{~N}$ ghép nối với Mass Selective Detector Agilent HP 5973 MSD. Cột HP-5MS có kích thước $0,25 \mu \mathrm{m}$ x $30 \mathrm{~m}$ x $0,25 \mathrm{~mm}$ và HP1 có kích thước $0,25 \mu \mathrm{m}$ x $30 \mathrm{~m} \times 0,32 \mathrm{~mm}$. Chương trình nhiệt độ với điều kiện $60^{\circ} \mathrm{C} / 2$ phút; tăng nhiệt độ $4^{\circ} \mathrm{C} / 1$ phút cho đến $220^{\circ} \mathrm{C}$, sau đó lại tăng nhiệt độ $20^{\circ} \mathrm{C} /$ phút cho đến $260^{\circ} \mathrm{C}$; với He làm khí mang. Việc xác nhận các cấu tử được thực hiện bằng cách so sánh các dữ kiện phổ MS của chúng với phổ chuẩn đã được công bố có trong thư viện Willey/Chemstation HP [14-17].

\section{Kết quả nghiên cứu}

Nghiên cứu thành phần hóa học của tinh dầu từ lá Trâm bullock (Syzygium bullockii) và lá 
Trâm quả trắng (Syzygium tsoongii) (Bảng 1), mẫu được thu ở Khu Bảo tồn thiên nhiên Kẻ Gỗ, tỉnh Hà Tĩnh. Hàm lượng tinh dầu đạt lần lượt là
$0,36 \%$ và $0,27 \%$ trọng lượng tươi, tinh dầu có màu vàng nhạt, được phân tích bằng sắc kí khí (GC) và sắc kí khí - khối phổ liên hợp (GC/MS).

Bảng 1. Thành phần hóa học tinh dầu lá loài Trâm bullock (Syzygium bullockii) và loài Trâm quả trắng (Syzygium tsoongii).

\begin{tabular}{|c|c|c|c|c|c|}
\hline \multirow{2}{*}{$\mathrm{TT}$} & \multirow{2}{*}{ Hợp chất } & \multirow{2}{*}{$\mathrm{RI}^{\mathrm{a}}$} & \multirow{2}{*}{$\mathrm{RI}^{\mathrm{b}}$} & \multicolumn{2}{|c|}{ Tỷ lệ \% } \\
\hline & & & & S.tsoongii & S.bullockii \\
\hline 1 & $\alpha$-pinene & 939 & 932 & - & 0.24 \\
\hline 2 & myrcene & 992 & 988 & 1.87 & - \\
\hline 3 & $(\mathrm{Z})-\beta$-ocimene & 1038 & 1034 & 10.61 & - \\
\hline 4 & (E)- $\beta$-ocimene & 1049 & 1044 & 4.99 & 0.17 \\
\hline 5 & $\gamma$-terpinene & 1063 & 1056 & 0.28 & - \\
\hline 6 & linalool & 1101 & 1100 & 0.30 & - \\
\hline 7 & allo-ocimene & 1131 & 1128 & 0.20 & - \\
\hline 8 & 2-undecanone & 1294 & 1294 & - & 0.99 \\
\hline 9 & $\delta$-elemene & 1348 & 1342 & 0.90 & - \\
\hline 10 & $\alpha$-copaene & 1389 & 1374 & 0.36 & - \\
\hline 11 & E-methyl cinnamate & 1390 & 1392 & 0.37 & 0.23 \\
\hline 12 & 2-dodecanone & 1396 & 1397 & - & 0.13 \\
\hline 13 & cis- $\beta$-elemene & 1403 & 1389 & 2.15 & 0.37 \\
\hline 14 & $\alpha$-gurjunene & 1425 & 1410 & 0.19 & 0.17 \\
\hline 15 & E-caryophyllene ( $=\beta$-caryophyllene) & 1439 & 1417 & 23.40 & 49.65 \\
\hline 16 & $\gamma$-elemene & 1444 & 1441 & 0.12 & - \\
\hline 17 & $\beta$-gurjunene (=calarene) & 1446 & 1445 & 0.12 & 0.18 \\
\hline 18 & aromadendrene & 1457 & 1440 & 0.60 & 1.01 \\
\hline 19 & cadina-3,5-diene & 1467 & 1458 & 0.12 & - \\
\hline 20 & $\alpha$-humulene & 1472 & 1452 & 6.33 & 2.78 \\
\hline 21 & 9-epi-(E)-caryophyllene & 1479 & 1465 & 1.15 & 1.03 \\
\hline 22 & trans-cadina-1(6),4-diene & 1488 & 1496 & 0.29 & - \\
\hline 23 & Unknown $(43,204$, RI 1488) & 1488 & - & - & 1.59 \\
\hline 24 & $\gamma$-muurolene & 1490 & 1501 & 0.35 & 0.69 \\
\hline 25 & $\alpha$-amorphene & 1494 & 1484 & 2.34 & - \\
\hline 26 & 2-tridecanone & 1497 & 1497 & - & 3.25 \\
\hline 27 & germacrene D & 1498 & 1485 & 2.52 & - \\
\hline 28 & 2-tridecanol & 1502 & 1502 & - & 1.67 \\
\hline 29 & $\beta$-selinene & 1504 & 1500 & 0.17 & - \\
\hline 30 & $\delta$-selinene & 1506 & 1506 & 0.17 & - \\
\hline 31 & $(\mathrm{E}, \mathrm{E})-\alpha$-farnesene & 1512 & 1505 & 3.59 & - \\
\hline 32 & viridiflorene & 1512 & 1505 & - & 0.28 \\
\hline 33 & bicyclogermacrene & 1516 & 1500 & 21.23 & 3.35 \\
\hline 34 & $\delta$-amorphene & 1522 & 1514 & 1.34 & 0.14 \\
\hline 35 & aromadendra-1(10),4(15)-diene & 1524 & - & - & 0.21 \\
\hline 36 & $\gamma$-cadinene & 1530 & 1515 & 0.44 & 0.16 \\
\hline 37 & $\delta$-cadinene & 1537 & 1522 & 2.77 & 0.65 \\
\hline 38 & zonarene & 1541 & 1530 & 0.30 & - \\
\hline 39 & trans-cadina-1,4-diene & 1547 & 1537 & 0.14 & - \\
\hline 40 & $\alpha$-cadinene & 1552 & 1537 & 0.17 & - \\
\hline 41 & (E)-nerolidol & 1569 & 1568 & - & 0.26 \\
\hline 42 & germacrene B & 1577 & 1584 & 0.25 & - \\
\hline
\end{tabular}




\begin{tabular}{|c|c|c|c|c|c|}
\hline 43 & palustrol & 1588 & 1581 & 0.29 & - \\
\hline 44 & spathulenol & 1596 & 1577 & 0.96 & 4.29 \\
\hline 45 & viridiflorol & 1604 & 1591 & 1.49 & - \\
\hline 46 & caryophyllene oxide & 1604 & 1581 & - & 4.14 \\
\hline 47 & cubeban-11-ol & 1613 & 1601 & 0.86 & 0.32 \\
\hline 48 & rosifoliol & 1621 & 1615 & 0.25 & - \\
\hline 49 & ledol & 1624 & 1638 & 0.16 & - \\
\hline 50 & torilenol & 1628 & - & - & 0.13 \\
\hline 51 & 5-guaiene-11-ol & 1642 & 1637 & 0.21 & - \\
\hline 52 & 1-epi-cubenol & 1646 & 1646 & 0.38 & - \\
\hline 53 & cubenol & 1651 & 1650 & 0.19 & - \\
\hline 54 & epi- $\alpha$-cadinol (=Tau-cadinol) & 1657 & 1656 & 1.10 & - \\
\hline 55 & epi- $\alpha$-muurolol (=T-muurolol) & 1659 & 1652 & 0.89 & - \\
\hline 56 & $\alpha$-muurolol $(=\delta$-cadinol $)$ & 1662 & 1662 & 0.49 & - \\
\hline 57 & $\alpha$-cadinol & 1672 & 1670 & 2.31 & - \\
\hline 58 & Unknown (79,220,RI 1672) & 1672 & - & - & 1.28 \\
\hline 59 & Unknown (43,224,RI 1675) & 1675 & - & - & 4.71 \\
\hline 60 & 14-hydroxy-9-epi-(E)-caryophyllene & 1688 & 1702 & - & 0.26 \\
\hline 61 & Unknown $(58,226$, RI 1700$)$ & 1700 & - & - & 3.47 \\
\hline 62 & Unknown $(79,248$, RI 1880) & 1880 & - & - & 1.25 \\
\hline 63 & Unknown $(91,246$, RI 2100$)$ & 2100 & - & - & 3.42 \\
\hline \multicolumn{4}{|c|}{ Tổng } & 99.71 & 92.47 \\
\hline \multicolumn{4}{|c|}{ Monoterpene hydrocarbon } & 18.25 & 0.41 \\
\hline \multicolumn{4}{|c|}{ Monoterpene có chứa oxy } & 0.37 & 6.27 \\
\hline \multicolumn{4}{|c|}{ Sesquiterpene hydrocarbon } & 71.51 & 60.67 \\
\hline \multicolumn{4}{|c|}{ Sesquiterpene có chứa oxy } & 9.58 & 9.40 \\
\hline \multicolumn{4}{|c|}{ Các hợp chất chưa xác định } & 0.0 & 15.72 \\
\hline
\end{tabular}

Từ lá Trâm bullock (Syzygium bullockii) đã định lượng được $92,47 \%$ tổng lượng tinh dầu, trong đó đã xác định được 27 hợp chất chiếm $76,75 \%$, còn 6 hợp chất chưa xác định tên chiếm $15,72 \%$. Trong tinh dầu được đặc trưng bởi các sesquiterpene hydrocarbon $(60,67 \%)$, sesquiterpenes có chứa oxy $(9,40 \%)$, monoterpene có chứa oxy $(6,27 \%)$, monoterpene hydrocarbon $(0,41 \%)$ và các thành phần chưa xác định tên chiếm $(15,72 \%)$. Thành phần chính của tinh dầu là E-caryophyllene $(49,65 \%)$, spathulenol (4,29\%), caryophyllene oxide $(4,14 \%)$, bicyclogermacrene $(3,35 \%), 2$ tridecanone $(3,25 \%), \alpha$-humulene $(2,78 \%)$, 9-epi(E)-caryophyllene $(1,03 \%)$ và aromadendrene $(1,01 \%)$.

Lá loài Trâm quả trắng (Syzygium tsoongii) đã xác định được 46 hợp chất chiếm $99,71 \%$ tổng lượng tinh dầu. Trong tinh dầu được đặc trưng bởi các sesquiterpene hydrocarbon (71,51\%), monoterpene hydrocarbon (18,25\%), sesquiterpenes có chứa oxy $(9,58 \%)$, monoterpene có chứa oxy $(0,37 \%)$ và các thành phần chưa xác định tên không có thành phần nào. Các hợp chất: E-caryophyllene $(23,40 \%)$, bicyclogermacrene $\quad(21,23 \%), \quad(\mathrm{Z})-\beta$-ocimene $(10,61 \%), \quad \alpha$-humulene $(6,33 \%), \quad(\mathrm{E})-\beta$-ocimene $(4,99 \%), \delta$-cadinene $(2,77 \%),(\mathrm{E}, \mathrm{E})-\alpha$-farnesene $(3,59 \%)$, germacrene D $(2,52 \%), \alpha$-amorphene $(2,34 \%), \quad \alpha$-cadinol $(2,31 \%)$ và cis- $\beta$-elemene $(2,15 \%)$ là các thành phần chính của tinh dầu, các hợp chất còn lại chiếm tỷ lệ không đáng kể.

Kết quả ở trên cho thấy tinh dầu của hai loài nghiên cứu đều được đặc trưng bỡi sesquiterpene hydrocarbon chiếm tỉ lệ lớn nhất trong tinh dầu. Thành phần chính trong tinh dầu của hai loài này đều chứa lượng lớn hợp chất E-caryophyllene. Tuy nhiên, đối với loài Trâm quả trắng (Syzygium tsoongii) còn được đặc trưng bỡi các monoterpene hydrocarbon chiếm $18,25 \%$, trong khi đó loài Trâm bullock (Syzygium bullockii) các hợp chất này 
chỉ chiếm $0,41 \%$. Thêm vào đó, loài Trâm quả trắng (Syzygium tsoongii) ngoài hợp chất chính là Ecaryophyllene $(23,40 \%)$ còn có một số hợp chất chiếm tỉ lệ cao trên $10 \%$ như bicyclogermacrene $(21,23 \%)$ và $(Z)-\beta$-ocimene $(10,61 \%)$, trong khi đó loài Trâm bullock không chứa hợp chất $(\mathrm{Z})-\beta$ ocimene, còn hợp chất bicyclogermacrene chiếm tỉ lệ thấp tương ứng 3,35\%. Đây là những dẫn liệu mới về tinh dầu của hai loài này.

\section{Kết luận}

Hàm lượng tinh dầu từ lá loài Trâm bullock (Syzygium bullockii) đạt 0,36\% trọng lượng tươi. 27 hợp chất được xác định chiếm $76,75 \%$, còn 6 hợp chất chưa xác định tên chiếm $15,72 \%$. Trong tinh dầu được đặc trưng bởi các hợp chất $\mathrm{E}$ caryophyllene $(49,65 \%)$, spathulenol $(4,29 \%)$ và caryophyllene oxide $(4,14 \%)$.

Loài Trâm quả trắng (Syzygium tsoongii) đạt 0,27\%, đã xác định được 46 hợp chất chiếm 99,71\% tổng lượng tinh dầu. E-caryophyllene $(23,40 \%)$, bicyclogermacrene $\quad(21,23 \%), \quad(\mathrm{Z})-\beta$-ocimene $(10,61 \%)$ và $\alpha$-humulene $(6,33 \%)$ là các thành phần chính của tinh dầu.

Đây là những dẫn liệu đầu tiên về thành phần hóa học trong tinh dầu lá của hai loài này.

\section{Tài liệu tham khảo}

[1] Z. Y. Wu, P. H. Raven, D. Y. Hong, Myrtaceae, J. Chen, L.A. Craven, Flora of China, Science Press, Beijing and Missouri Botanical Garden Press, St Louis, Missouri, 2007, pp. 321-359.

[2] K. Larsen, T. Santisuk, Myrtaceae, J. Parnell, Chantaranothai, Flora of Thailand, The Forest Herbarium, Bangkok Publishing, 2002, pp. 778914.

[3] R. Govaerts, M. Sobral, P. Ashton, F. Barrie, B. K. Holst, L. L. Landrum, K. Matsumoto, F. F. Mazine, E. N. Lighadha, C. Proenca, L.H. SoaresSilva, P.G. Wilson, E. Lucas, World Checklist of Myrtaceae, Royal Botanic Gardens Richmond, UK, 2008.

[4] W. K. Soh, J. Parnell, A Revision of Syzygium Gaertn. (Myrtaceae) in Indochina (Cambodia,
Laos and Vietnam), Adansonia, Vol. 37, No. 2, 2015, pp. 179-275.

https://doi.org/10.5252/a2015n2a1.

[5] N. T. Ban, Checklist of Plant Species of Vietnam, Agriculture Publishing House, Hanoi, 2003 (in Vietnamese).

[6] P. H. Ho, An Illustrated Flora of Vietnam, Youth Publishing House, Ho Chi Minh, 2003 (in Vietnamese).

[7] V. V. Chi, The Dictionary of Medicinal Plants of Vietnam, Medical Publishing House, Hanoi, 2018 (in Vietnamese).

[8] D. T. Loi, Medicinal Trees and Medicaments of Vietnam, Medical Publishing House, Hanoi, 2004 (in Vietnamese).

[9] L. D. Moi, L. D. Cu, T. M. Hoi, T. H. Thai, N. K. Ban, Essential Oil Plant Resources in Vietnam, Agriculture Publishing House, Hanoi, 2000 (in Vietnamese).

[10] A. Hamad, M. G. P. Mahardica, I. Yuliani, Hartanti, Chemical Constituents and Antimicribial Activities of Essential Oils of Syzygium Polyanthum and Syzygium Aromaticum, Rasayan Journal of Chemistry, Vol. 10, No. 2, 2017, pp. 564-569. http://dx.doi.org/10.7324/RJC.2017.1021693.

[11] D. H. Bich, Medicinal Plants and Medicinal Animals in Viet Nam, Science and Technology Publishing House, Hanoi, 2006 (in Vietnamese).

[12] T. H. Khanh, P. H. Ban, Analysis of Essential Oils from Leaf of Syzygium Hancei Merr. \& Perry, Syzygium caryophyllatum (L.) Alston and Syzygium lineatum (DC.) Merr. \& Perry from Vietnam, Journal of Essential Oil Bearing Plants, Vol 23, No. 3, 2020 pp. 548-558. https://doi.org/10.1080/0972060X.2020.1790429.

[13] Ministry of Health, Vietnamese Pharmacopoeia, The Medical Publishing House, Hanoi, 2010 (in Vietnamese).

[14] R. P. Adams, Identification of Essential Oil Components by Gas Chromatography/ Quadrupole Mass Spectrometry, Allured Publishing Corp, Carol Stream, IL, 2001.

[15] S. R. Heller, G. W. A. Milne, EPA/NIH Mass Spectral Data Base, U.S. Government Printing Office, Washington DC, USA, 1983.

[16] E. Stenhagen, S. Abrahamsson, F. W. McLafferty, Registry of Mass Spectral Data, Wiley, New York, 1974.

[17] NIST Chemistry WebBook, NIST Standard Reference Database Number 69. https://webbook.nist.gov/chemistry/, 2020 (accessed 1 August 2020). 